\title{
Neutrophil NETs in reproduction: from infertility to preeclampsia and the possibility of fetal loss
}

\author{
Sinuhe Hahn ${ }^{1 *}$, Stavros Giaglis ${ }^{1,2 *}$, Irene Hoesli ${ }^{3}$ and Paul Hasler ${ }^{2}$ \\ ${ }^{1}$ Department of Biomedicine, University of Basel/Laboratory for Prenatal Medicine, University Clinics, University Women's Hospital, Basel, Switzerland \\ 2 Department of Rheumatology, Cantonal Hospital Aarau, Aarau, Switzerland \\ ${ }^{3}$ Department of Obstetrics, University Clinics, University Women's Hospital, Basel, Switzerland
}

\section{Edited by:}

Marko Radic, University of

Tennessee, USA

Reviewed by:

lan L. Sargent, University of Oxford,

UK

Christian C. Yost, University of Utah

School of Medicine, USA

Amy C. Hermesch, University of

Illinois at Chicago, USA

\section{*Correspondence:}

Sinuhe Hahn and Stavros Giaglis,

Department of Biomedicine,

University of Basel/Laboratory for

Prenatal Medicine, University

Clinics, University Women's

Hospital, Hebelstrasse 20, CH 4031 ,

Basel, Basel-Stadt, Switzerland.

e-mail:shahn@uhbs.ch;

stavros.giaglis@unibas.ch
The intention of this review is to provide an overview of the potential role of neutrophil extracellular traps (NETs) in mammalian reproduction. Neutrophil NETs appear to be involved in various stages of the reproductive cycle, starting with fertility and possibly ending with fetal loss. The first suggestion that NETs may play a role in pregnancy-related disorders was in preeclampsia, where vast numbers were detected in the intervillous space of affected placentae. The induction of NETosis involved an auto-inflammatory component, mediated by the increased release of placental micro-debris in preeclampsia. This report was the first indicating that NETs may be associated with a human pathology not involving infection. Subsequently, NETs have since then been implicated in bovine or equine infertility, in that semen may become entrapped in the female reproductive tract during their passage to the oocyte. In this instance interesting species-specific differences are apparent, in that equine sperm evade entrapment via expression of a DNAse-like molecule, whereas highly motile bovine sperm, once free from seminal plasma (SP) that promotes interaction with neutrophils, appear impervious to NETs entrapment. Although still in the realm of speculation it is plausible that NETs may be involved in recurrent fetal loss mediated by anti-phospholipid antibodies, or perhaps even in fetal abortion triggered by infections with microorganisms such as $L$. monocytogenes or B. abortus.

Keywords: pregnancy, preeclampsia, infertility, recurrent fetal loss, neutrophil extracellular traps (NETs)

\section{INTRODUCTION}

Polymorphonuclear neutrophils (PMNs) are the most prevalent leucocyte cell type in the peripheral circulation. They are characterized by a uniquely lobulated nuclear structure and a highly granulated cytoplasm. PMNs play a leading role in combatting infection either by phagocytosis or by the release of antibacterial granules (Nathan, 2006). A more recent attribute of these cells is their ability to extrude their DNA into the extracellular environment, as so-called neutrophil extracellular traps (NETs), which serve to ensnare and kill bacteria (Brinkmann et al., 2004; Brinkmann and Zychlinsky, 2007). This unique form of cell death termed NETosis has been shown to be highly reliant on the action of nicotinamide adenine dinucleotide phosphate (NADPH) oxidase, the generation of reactive oxygen species (ROS) (Fuchs et al., 2007), and the combined actions of myeloperoxidase (MPO), neutrophil elastase (NE) (Papayannopoulos et al., 2010), and histone deamination by human peptidylarginine deiminase 4 (PAD4) (Neeli et al., 2008; Wang et al., 2009). It is this facet which forms the basis of this review.

PMNs are involved in several stages in the reproductive cycle. In many mammalian species, PMNs have been implicated

Abbreviations: FRT, female reproductive tract; PMN, polymorphonuclear neutrophil; MC, menstrual cycle; SP, seminal plasma; AI, artificial insemination; IUGR, intra-uterine fetal growth restriction; NE, neutrophil elastase; aPL, antiphospholipid antibodies; $\beta 2 \mathrm{GPI}$, beta-2-glycoprotein I; PAR-2, protease-activated receptor 2 ; ROS, reactive oxygen species. in tissue remodeling during the oestrous cycle when the endometrium adapts to be receptive for oocyte implantation (Strzemienski, 1989; Wood et al., 2007).

In murine systems, the influx of PMNs into the vaginal vault appears to play a crucial role in the continued progression of the oestrous cycle, as their depletion via application of antiGr-1 monoclonal antibodies leads to a blocking of the cycle in diestrus (Sasaki et al., 2009). This blockage was reversible, and could be reverted by the re-introduction of circulatory PMNs into affected mice. This neutropenic abrogation of the oestrous cycle was accompanied by reduction in the levels of the sex hormones oestradiol and progesterone, which are necessary for its maintenance, implying some form of feedback mediated by normal endometrial presence of infiltrating PMNs. The feedback loop was facilitated by opioid peptides released by activated PMNs, which promote steroidogenesis by granulosa cells (Sasaki et al., 2011).

During the human menstrual cycle (MC), large numbers of PMNs have been noted in areas of tissue degradation (day 28) prior to onset of menstruation (Salamonsen and Lathbury, 2000). It appears that endometrial PMNs may have different phenotypes, as not all stained positive for metalloproteinase- 9 (MMP-9) (Salamonsen et al., 2000). Endometrial PMNs have also been determined to express ELAFIN, a serine protease inhibitor and member of the whey acidic protein (WAP) family (King et al., 2003a,b). Elafin is a potent inhibitor of NE 
and proteinase 3, and has been suggested to protect tissue from degradation by these enzymes (King et al., 2003a,b). Since Elafin has been demonstrated to possess anti-microbial activity, its presence could thereby contribute to innate immune defence in the female reproductive tract (FRT) (King et al., 2003a,b).

Hormonal changes occurring during the human MC have also been shown to alter the physiology of circulating PMNs. During the luteal phase of the $\mathrm{MC}$, in which progesterone peaks, a notable increase in circulatory PMNs numbers was noted (Smith et al., 2007). Circulatory PMNs numbers remained high until the onset of menstruation at day 28, at which time they were still higher than those of comparable male donors. During the luteal phase circulatory PMNs exhibited reduced levels of MMP-9 and TNF- $\alpha$ expression (Smith et al., 2007). During periods of oestrogen surges (week 2 and 3 of the MC), circulatory PMNs in women expressed lower levels of CD89 (FcaR for IgA), CD11b, and CD18, which returned to normal by week 4 . In general, circulatory PMNs in women expressed higher levels of CD89 than those from men (Smith et al., 2007).

The reduction in expression of the integrins CD11b and CD18 could explain the diminution of neutrophil adhesion by oestrogen, and partially explain the anti-inflammatory action of this sex hormone. The generally elevated expression of CD89 on peripheral PMNs by women indicates that they are more responsive to activation via an IgA-based mechanism. Women mostly exhibit a more robust humoral response than men.

In animal systems it is unclear if the oestrus cycle affects circulatory and FRT-associated PMN pro-inflammatory activity. In hormone primed mares no change in the bactericidal activity of circulatory PMNs was noted (Strzemienski et al., 1987).

\section{INTERACTION BETWEEN NEUTROPHILS AND SEMEN IN THE FEMALE REPRODUCTIVE TRACT}

During coitus billions of sperm are deposited into the FRT. During the sexual act, microorganisms originating from either the penis or the vagina are transported with the ejaculate either into the vagina or directly into the uterus. In order to maintain an environment favorable to implantation, these potential pathogens need to be successfully eliminated. A further task is the removal of the vast majority of sperm, as these can be antigenic to the recipient female, and immunization against sperm can result in infertility. A number of observations have shown that neutrophils are recruited to the FRT following insemination in a manner akin to inflammation, and that they play a major role in the removal of excess sperm, largely via phagocytosis (Strzemienski, 1989; Alghamdi and Foster, 2005; Alghamdi et al., 2009; Katila, 2012).

Even though human reproductive tract leucocytosis has been implicated in human infertility, interactions between neutrophils and sperm have been best studied in large domesticated animals such as cows and horses, due to the frequent use of artificial insemination (AI) for optimal breeding (Katila, 2012). In these instances it has been observed that the repeated deposition of spermatozoa in the presence of neutrophils can lead to diminished fertility (Alghamdi and Foster, 2005; Alghamdi et al., 2009).

In early studies it was noted that bovine seminal plasma (SP) reduced the ability of isolated PMNs to phagocytize bull spermatozoa (Strzemienski, 1989). In further exploratory studies examining the action of SP it was evident that equine SP contained factors that reduced neutrophil binding to spermatozoa in vitro (Alghamdi et al., 2004), thereby perhaps permitting a greater number of healthy mobile spermatozoa to reach the oviduct.

In these studies aggregates were noted between large numbers of PMNs and spermatozoa, which could be antagonized by SP. The issue of these PMN-spermatozoa aggregates was subsequently addressed in more detail once it emerged that PMNs were capable of producing extracellular traps (Brinkmann et al., 2004).

Since bovine SP was found to contain a fertility-promoting factor with homology to DNAse I, the question was raised whether such a factor would permit spermatozoa to evade the presence of any PMN NETs in the FRT. In one of the first publications recording the presence of NETs in another system than infection, Alghamdi and colleagues observed that the incubation of isolated peripheral PMNs with equine spermatozoa lead to the vigorous generation of NETs, with kinetics close to those mediated by E. coli (Alghamdi and Foster, 2005). They furthermore observed that the protein fraction of equine SP did indeed contain a molecule with DNAse activity, as it was capable of digesting plasmid DNA, in a manner very similar to that performed by DNAse I (Alghamdi and Foster, 2005).

The addition of this equine SP protein fraction to spermatozoa-PMN mixtures led to the digestion of PMN NETs, an aspect that could be partially mimicked by the addition of extraneous DNAse I. It was, however, clear that equine SP contains other factors that modulate PMNs response to spermatozoa, as it reduced the number of NETs generated by accessory PMNs in such cultures (Alghamdi and Foster, 2005) (Figure 1).

Of great interest is that equine SP protein fraction did not prevent NETs induction by E. coli, nor did it prevent their killing by activated PMNs. Of further note is that this equine DNAse-like protein was virtually absent in the SP of a stallion with a record of poor fertility. This strongly suggests that this factor assists in mediating optimal interaction between spermatozoa and PMN in the equine FRT, without compromising PMN bacteriocidal activity (Alghamdi and Foster, 2005).

A major difference between bovine and equine insemination is that in the former the spermatozoa are deposited in the vagina, whilst in the latter they are ejaculated directly into the uterus (Alghamdi et al., 2009; Katila, 2012). In bovines the spermatozoa need to migrate through the cervix into the uterus, in the process leaving most of the seminal fluid behind. Therefore, the interaction between SP proteins modulating PMN activity will be diminished in this case. Alternatively, in equines, SP proteins could have a different function, because spermatozoa are discharged adjacent to the site of fertilization.

This indeed turned out to be the case in that bovine SP mediates a different modality on PMN activity than equine SP, by promoting the binding of bovine spermatozoa to PMNs (Alghamdi et al., 2009). On the other hand, in contrast to the situation in 


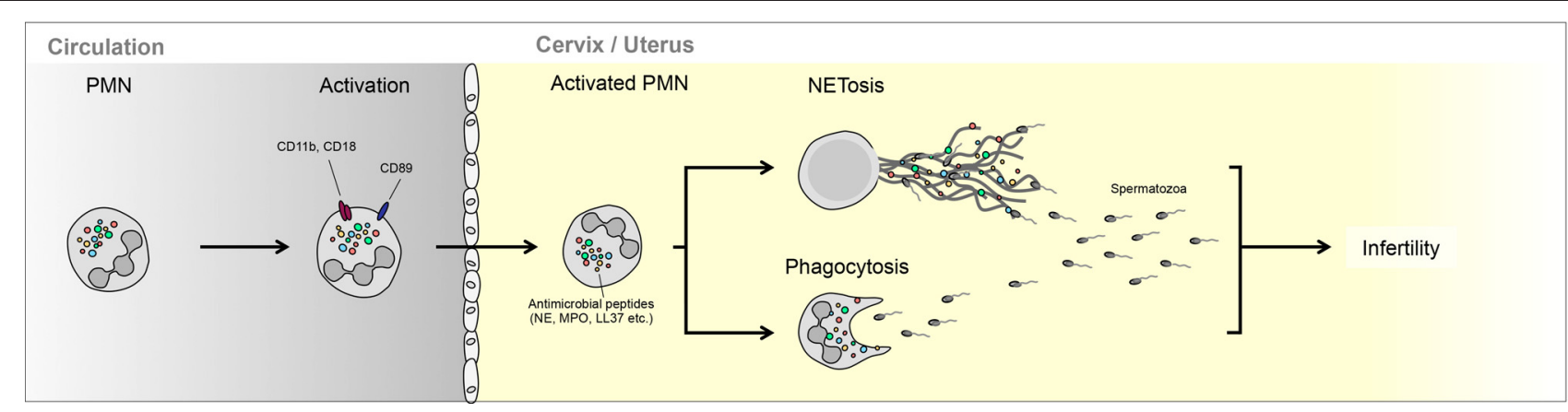

FIGURE 1 | Interaction between neutrophils and semen in the female reproductive tract. PMN can either phagocytize less motile spermatozoa or trap these in NETs. The ability of PMNs to interact with spermatozoa is regulated largely by seminal plasma, which can promote NETting (bovine) or enhance escape (equine).

horses, bovine spermatozoa cleared completely of SP showed low binding affinity to PMN, for a period as long as $3 \mathrm{~h}$. This implies that less mobile bovine spermatozoa will be ensnared by NETs directly in the bovine vagina, due to the action of SP, while virile highly motile spermatozoa will enter the uterus cleared of SP and thereby evade interaction with any PMNs present in this part of the FRT (Alghamdi et al., 2009).

Apart from demonstrating remarkable species-specific differences, these results have major implications in how AI is carried out, and under which conditions SP protein fractions may have beneficial character or not (Alghamdi et al., 2009; Katila, 2012).

\section{OCCURRENCE OF NETS IN PREECLAMPSIA}

Although the vast majority of human pregnancies progress to term without any complications, many can be affected by disorders such as fetal growth restriction, preeclampsia, or preterm labor, which can result in maternal or fetal mortality/morbidity (Wilkinson, 2011). To a large extent these different pathologies may hold their origin in placental abnormalities (Brosens et al., 2011).

Human placentation is unique by the depth of invasion of the maternal endometrium by fetal tissues, but also by the extent in which maternal blood vessels are modified by fetal trophoblast (Huppertz, 2008; Pijnenborg et al., 2011). In this manner, toward the end of the first trimester, extravillous trophoblast cells enter the maternal endometrium and replace the endothelial cells of the maternal spiral arteries (Redman, 1997). This leads to a significant enlargement and relaxation of these vessels, thereby ensuring an optimal blood flow though the intervillous space.

Failure of such a modification is associated with either severe intra-uterine fetal growth restriction (IUGR) and/or early onset preeclampsia (Redman and Sargent, 2005; Huppertz, 2008; Brosens et al., 2011).

Preeclampsia is a disorder unique to human pregnancy, characterized by proteinuria and sudden elevation in blood pressure in previously normotensive pregnant women (Roberts and Redman, 1993). Currently the only effective therapy is delivery of the baby, which frequently results in extreme prematurity, as the onset of this disorder can occur as early as 20 weeks of gestation. If left untreated, preeclampsia can develop into eclampsia, a potentially lethal condition, characterized by epilepsy-like convulsions (Roberts and Redman, 1993).
Even though the placenta plays a central role in the underlying aetiology (Huppertz, 2008), preeclampsia is associated with systemic damage of the maternal endothelium and an anomalous maternal inflammatory response (Redman and Sargent, 2010). This inflammatory response is mediated in part by the release of placental micro-debris by the placental syncytiotrophoblast, the deportation of which is elevated in cases with preeclampsia (Gupta et al., 2005b; Hahn et al., 2005; Germain et al., 2007; Messerli et al., 2010).

With regard to PMNs, previous studies have shown that normal human pregnancy is associated with a pro-inflammatory phenotype of these cells, a feature which is significantly more pronounced in preeclampsia (Sacks et al., 1998). This was reflected by the elevated expression of cell surfaces markers such as CD11b, but also intracellular activation markers, such as iROS (Sacks et al., 1998). Additional evidence of peripheral PMN activation under these conditions is provided by the presence of elevated levels of NE in the plasma of patients with preeclampsia (Halim et al., 1996; Gupta et al., 2006b). This activation appears to be stimulated by the presence of placental micro-debris in the maternal circulation (Aly et al., 2004).

Prompted by our observations that preeclampsia was associated with significantly elevated fetal and maternal cellfree DNA in the maternal circulation (Zhong et al., 2001b, 2005a,b), we were very intrigued by the report that PMNs can release their nuclear DNA into the extracellular environment (Brinkmann et al., 2004). Consequently, we set out to determine whether a possible connection existed between these two phenomena.

For this purpose we used in vitro co-cultures using peripheral PMNs isolated from healthy controls and highly purified placental micro-debris (Gupta et al., 2005a). In our experiments we observed that placental micro-debris led to the activation of PMN as assessed by the elevated expression of CD11b (Gupta et al., 2005a). This activation by placental micro-debris was accompanied by the generation of NETs, in a time and dose dependent manner (Gupta et al., 2005a), with similar kinetics to what had been previously observed using bacterial agents (Brinkmann et al., 2004).

We also observed that NETs could be induced by other placentally derived factors, such as the cytokine IL-8. It is therefore possible that placentally derived micro-debris and inflammatory 
cytokines (IL-8) may act in concert in the activation of PMNs and induction of NETs in pregnancy (Gupta et al., 2005a).

To assess whether these in vitro observations had any physiological relevance we examined placentae from normal healthy term deliveries or those affected by severe preeclampsia. PMN NETs could be detected in the intervillous space of normal placentae. This is to be expected as the normal placenta does deport micro-debris, which could lead to PMNs activation and ensuing NETosis, as part of the pro-inflammatory condition observed in normal pregnancy. The number of NETs in preeclamptic placentae was, however, dramatically elevated and appeared to fill the entire intervillous space in certain instances.

As preeclampsia is characterized by hypoxia-reperfusion damage (Burton and Jauniaux, 2004), the presence of large numbers of NETs directly in the intervillous space, the site of oxygen exchange between mother and fetus, may contribute to this.

Since NETs have recently been shown to promote thrombi in deep vein thrombosis (DVT) and in tumor model systems (Brill et al., 2012; Demers et al., 2012; Fuchs et al., 2012), it is possible that the occlusion of blood flow through the intervillous space by NETs could be exacerbated by such an additional event. The likelihood of such an event is high, as excessive fibrin deposition and infarction are frequently observed in preeclamptic placentae (Kitzmiller and Benirschke, 1973).

PMN NETs may also contribute to the widespread systemic damage to the maternal endothelium observed in preeclampsia (Powe et al., 2011), since endothelial cells are susceptible to cell death induced by NETting PMNs (Gupta et al., 2010).

Additional support suggesting that placentally derived factors contribute to PMN activation in preeclampsia is provided by the study of circulatory PMNs isolated from maternal blood isolated from the antecubital and uterine veins (Mellembakken et al., 2002). In cases with preeclampsia, it was observed that PMNs passing through the uteroplacental circulation appeared to have a more highly activated phenotype than those present in the peripheral circulation. No similar alteration was observed in normal pregnancies. These data therefore suggest that an inflammatory process occurs in the decidua and placental tissues during the development of preeclampsia (Mellembakken et al., 2002) (Figure 2).

It is currently unclear whether NETs represent an initiating lesion in preeclampsia, or are the result of another underlying placental deficiency. By using the data on maternal and fetal cell-free DNA levels in preeclampsia, it may be possible to tentatively infer regarding the time-point of NETs induction
(Gupta et al., 2006a). In this context, elevations in cell-free fetal DNA have been suggested to be the result of a placental lesion, whilst elevations in maternal cell-free DNA could result from the generation of NETs (Gupta et al., 2006a). A number of studies have indicated that disturbances in cell-free fetal DNA levels occur early in gestation, prior to the onset of preeclampsia symptoms (Leung et al., 2001; Zhong et al., 2001a). In contrast, cell-free maternal DNA is only elevated once the symptoms become manifest (Zhong et al., 2001a). These data imply that two phases are present in preeclampsia; a pre-clinical phase involving a placental lesion, and a second clinical phase with symptoms involving a systemic inflammatory response by the mother (Roberts and Hubel, 2009). It would, hence, appear that NETs are associated with the manifest disorder, and not a preclinical initiating lesion (Gupta et al., 2006a).

NETs also do not appear to distinguish between early and late onset preeclampsia, as both seem to be associated with increased PMNs activity as assessed by increased levels of NE in maternal plasma in both forms, when compared to healthy pregnancies (Gupta et al., 2006b).

Currently it is still not clear whether PMN NETs are involved in other pregnancy-related disorders such as IUGR, recurrent fetal loss or preterm labor (Hahn et al., 2006). It is also evident that the underlying aetiology of multi-factorial syndromes, such as preeclampsia, will not involve a single lesion, but rather involve a number of different triggering events (Redman and Sargent, 2005; Brosens et al., 2011). These may include the imbalance of placentally produced angiogenic factors (Powe et al., 2011; Rana et al., 2012), or the dysregulation of inflammatory cytokine networks (Rusterholz et al., 2007; Granne et al., 2011).

\section{A POSSIBLE ROLE OF NETS IN SPONTANEOUS FETAL LOSS}

Although there is no direct evidence that NETs may be implicated in fetal loss, induced either via the presence of autoantibodies or infectious agents, there is accruing evidence that PMNs activation may play a crucial part in these events (Weiler, 2008; Lynch and Salmon, 2010; Girardi, 2011). Recurrent fetal loss is frequently associated with the presence of maternal antiphospholipid antibodies (aPL) (Weiler, 2008; Lynch and Salmon, 2010; Girardi, 2011). aPL via the interaction with beta-2-glycoproteinI ( $\beta 2 \mathrm{GPI})$, binds to phosphotidylserine moieties on the trophoblast, thereby providing a key step for the activation of a series of coagulation factors, hence, the layman's term "sticky blood syndrome."

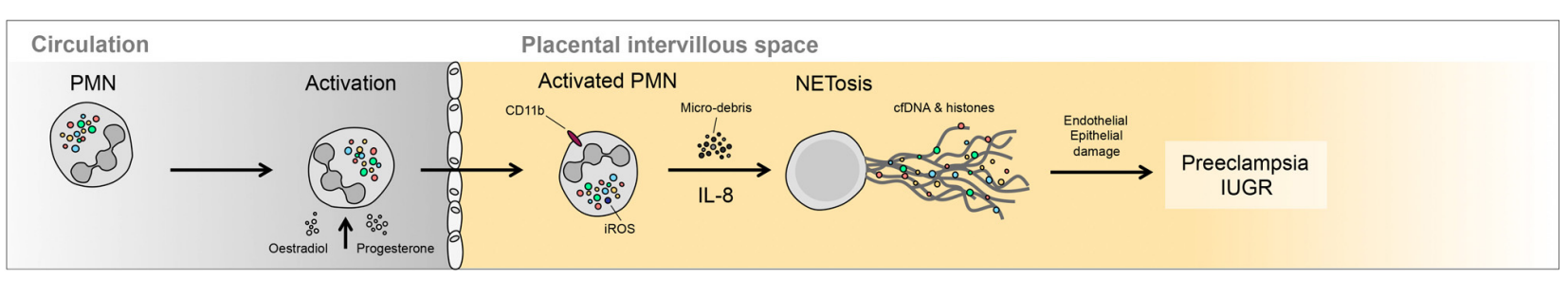

FIGURE 2 | Occurrence of NETs in preeclampsia. Large numbers of NETs have been detected directly in the intervillous space of preeclamptic placentae. These NETs appear to be triggered by the elevated release of highly inflammatory placental micro-debris. 
The traditional view is that the presence of the aPL leads to activation of the clotting cascade, thereby leading to placental infarction and ensuing fetal demise (Weiler, 2008; Lynch and Salmon, 2010; Girardi, 2011). Support for this notion is provided by the clinical use of low molecular weight heparin, which has been shown to be effective in preventing fetal loss in most cases (Hahn et al., 2006).

Recent evidence indicating that aPL is associated with an inflammatory activation of PMNs via the complement system is leading to a change of the traditional dogma (Salmon and Girardi, 2008).

Evidence for such an interaction was largely provided by murine model systems. It was determined that when aPL were infused into pregnant mice, fetal demise was not associated with a deposition of fibrin or increased presence of thrombi, but rather that it involved the activation of the complement system, in particular components $\mathrm{C} 3$ and $\mathrm{C} 5$, and the repressive activity of Crry (Holers et al., 2002; Girardi et al., 2003). In addition, the innate arm of the immune system was implicated, as the decidua of treated mice exhibited considerable PMNs infiltration and elevated tissue factor (TF) expression (Redecha et al., 2007).

These findings were largely achieved via the dissection of the underlying molecular pathways using a series of knock-out murine models or pharmacologic inhibitors. In these examinations it was determined that elevated TF expression by PMNs appeared to be a crucial component in triggering fetal demise, which was the result of a complex chain of events (Redecha et al., 2007). The first of these was binding of aPL to the trophoblast, thereby catalyzing zymogenic cleavage of C5. This permits the active component C5a to bind to the C5aR on PMNs. This receptor-ligand interaction triggers increased cell surface expression of TF, itself a receptor for coagulation factors FVIIa and FXa. Such receptor TF/FVIIa-FXa complexes lead to activation of the G-protein coupled protease-activated receptor 2 (PAR-2), which in PMNs initiate ROS production and the release of pro-inflammatory cytokines (Redecha et al., 2007; Weiler, 2008; Girardi, 2011).

PMNs appear to be the final effector in this complementtriggered cascade, as their depletion via the use of appropriate antibodies effectively blocks aPL induced fetal loss (Pierangeli et al., 2005). For this reason it was suggested aPL induced fetal loss was the result of uncontrolled ROS production by complement activated PMNs in the feto-maternal interface, leading to oxidative damage of underlying placental tissue (Salmon and Girardi, 2008).

As the production of ROS is a vital component of the pathway triggering NETosis and the release of DNA into the extracellular environment (Fuchs et al., 2007), it is enticing to speculate that NETs may occur in aPL induced fetal loss. Furthermore, as the presence of NETs can be cytotoxic to closely adjacent cells (Gupta et al., 2010), it is possible that the occurrence of such entities can contribute to trophoblast injury apparent in this disorder.

An interesting observation is that PMNs from preterm infants or neonates do not appear capable of undergoing NETosis, or at least showed a very delayed response, when treated with potent stimuli such as platelet activating factor (PAF) and lipopolysaccharide (LPS), unlike normal adult PMNs (Marcos et al., 2009; Yost et al., 2009). This facet did not appear to involve a defect in signaling pathways, as neonatal PMNs expressed the required receptors (PAF-R and TLR4) and displayed normal responses, such as calcium mobilization, production of IL-8 in response to these stimuli. This deficit could also not be overcome by supplementation of intracellular ROS pools by treatment with glucose/glucose oxidase. It is not clear from this particular study how representative the relatively inert nature of neonatal PMN in cases with preterm delivery is, since previous reports have suggested that intrauterine activation of PMNs occurs under these conditions, leading to pulmonary haemorrhage in affected infants (Mehta and Petrova, 2006).

Although it may seem that the coagulation cascade is not directly involved in this particular model system, since fetal loss could not be hindered by anti-clotting agents such as hirudin, which prevent thrombus formation but not complement activation, it may nevertheless play an accessory role. This would especially be the case if NETs were implicated in aPL induced fetal loss, as these could provide the necessary stimulus and scaffold for clot formation (Fuchs et al., 2012). This supposition is based on recent data indicating that NETs stimulate the extrinsic and intrinsic coagulation pathways, by promoting platelet and $\mathrm{RBC}$ adhesion and by concentrating effector proteins and coagulation factors involved in hemostasis (Fuchs et al., 2010, 2012; Massberg et al., 2010; von Bruhl et al., 2012). In this context NETs have been found to be abundant in experimental DVT in baboons and mice, co-localizing with vWF, an important endothelial clotting factor (Brill et al., 2012). Apart from providing a DNA-based scaffold facilitating the binding of pro-coagulatory factors such as Factor XII, intrinsic PMN derived serine proteases such as NE and cathepsin $\mathrm{G}$ can stimulate clotting via the cleavage of coagulation mediators such as tissue factor pathway inhibitor (TFPI), or fibrin (Plow, 1980; Higuchi et al., 1992; Massberg et al., 2010; Semeraro et al., 2011).

Given that preeclampsia, IUGR and even fetal loss are broadly related to dysfunctions at the interface between innate immunity and haemostasis, it would be of cardinal importance to investigate the potential triggers of PMNs activation and NETosis in these pathologies. The trigger could be the interaction of neutrophils with activated cells, such as platelets or endothelium (Gupta et al., 2010; Saffarzadeh et al., 2012), or alternatively, involve hypoxia, inflammatory cytokines, or factors generated early in thrombotic events (Fuchs et al., 2007).

The scope of PMN activity associated with fetal loss may, however, be even broader. This is based on recent data suggesting that infections with Brucella abortis (Gupta and Bianchi, 1997) or Listeria monocytogenes (Knowles et al., 2011) leads to PMNs recruitment and activation, including release of IL- 8 and ROS production. As brucellosis in cattle or listeriosis in humans can be directly associated with spontaneous abortion (Robbins and Bakardjiev, 2012), it is open to speculation whether NETs occur in infected placentae in these conditions, and thereby contribute to the process of fetal loss (Figure 3).

\section{CONCLUSIONS}

The activity of PMNs is modulated or altered during various phases of the reproductive cycle (Salamonsen and Lathbury, 2000; 


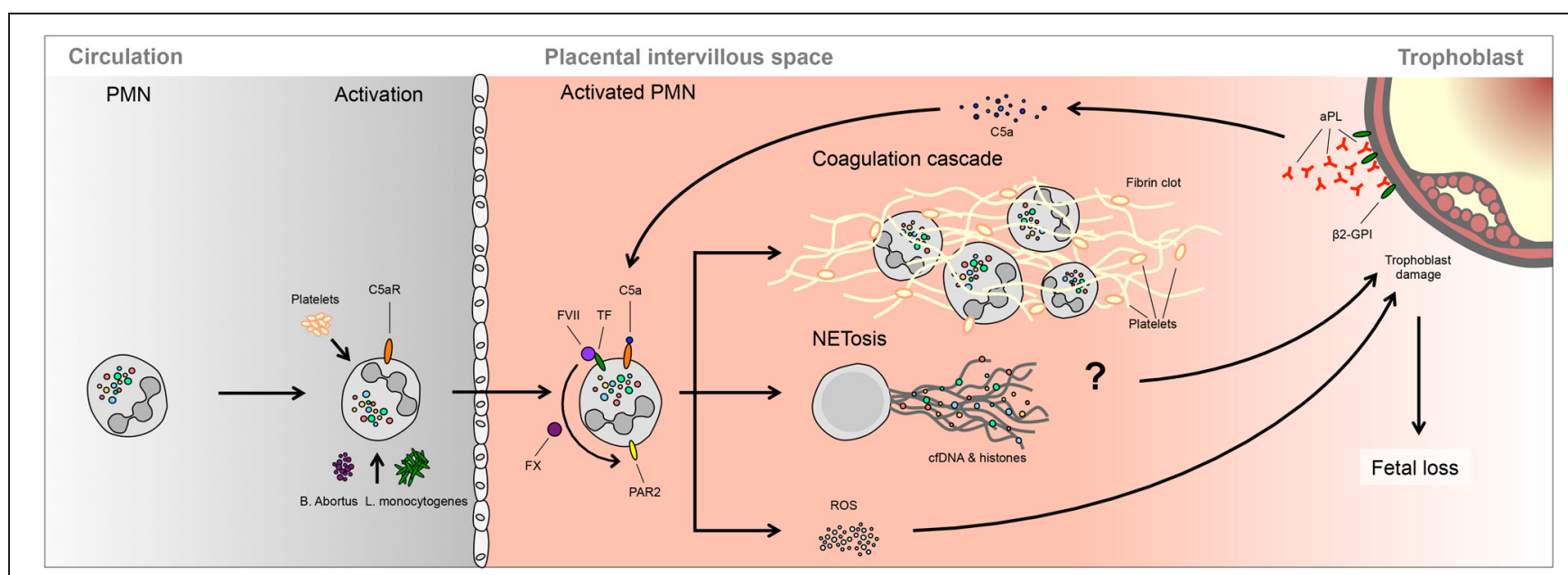

FIGURE 3 | A possible role of NETs in spontaneous fetal loss. In murine models, fetal loss triggered by $\mathrm{APL}$ involves activation of the complement cascade, notably $\mathrm{C} 3$ and $\mathrm{C} 5$, which lead to PMNs activation via TF. Ensuing ROS production is proposed to lead to trophoblast damage, leading to fetal demise. It is not clear whether this process involves NETosis or PMNs activation of the clotting cascade.

Wood et al., 2007; Sasaki et al., 2009). This is most evident in the menstrual or oestrum cycle, in which the activity of circulatory PMNs is altered by the action of sex hormones, but also in the endometrial tissue, where PMNs play a role in tissue removal or modification. PMNs appear to play a significant role in post mating inflammatory response (Katila, 2012), as well as in the trapping and clearance of sperm by NETs (Alghamdi et al., 2004; Alghamdi and Foster, 2005). In these instances, the action of seminal fluid reveals interesting species specific differences, in that in horses it down-modulates PMN activity, thereby promoting sperm escape, whereas in cattle it promotes

\section{REFERENCES}

Alghamdi, A. S., and Foster, D. N. (2005). Seminal DNase frees spermatozoa entangled in neutrophil extracellular traps. Biol. Reprod. 73, 1174-1181.

Alghamdi, A. S., Foster, D. N., and Troedsson, M. H. (2004). Equine seminal plasma reduces sperm binding to polymorphonuclear neutrophils (PMNs) and improves the fertility of fresh semen inseminated into inflamed uteri. Reproduction 127, 593-600.

Alghamdi, A. S., Lovaas, B. J., Bird, S. L., Lamb, G. C., Rendahl, A. K., Taube, P. C., et al. (2009). Species-specific interaction of seminal plasma on sperm-neutrophil binding. Anim. Reprod. Sci. 114, 331-344.

Aly, A. S., Khandelwal, M., Zhao, J., Mehmet, A. H., Sammel, M. D., and Parry, S. (2004). Neutrophils are stimulated by syncytiotrophoblast microvillous membranes to generate superoxide radicals in women with preeclampsia. Am. J. Obstet. Gynecol. 190, 252-258.
Brill, A., Fuchs, T. A., Savchenko, A. S., Thomas, G. M., Martinod, K., De Meyer, S. F., et al. (2012). Neutrophil extracellular traps promote deep vein thrombosis in mice. J. Thromb. Haemost. 10, 136-144.

Brinkmann, V., Reichard, U., Goosmann, C., Fauler, B., Uhlemann, Y., Weiss, D. S., et al. (2004). Neutrophil extracellular traps kill bacteria. Science 303, 1532-1535.

Brinkmann, V., and Zychlinsky, A. (2007). Beneficial suicide: why neutrophils die to make NETs. Nat. Rev. Microbiol. 5, 577-582.

Brosens, I., Pijnenborg, R., Vercruysse, L., and Romero, R. (2011). The "Great Obstetrical Syndromes" are associated with disorders of deep placentation. Am. J. Obstet. Gynecol. 204, 193-201.

Burton, G. J., and Jauniaux, E. (2004). Placental oxidative stress: from miscarriage to preeclampsia. J. Soc. Gynecol. Investig. 11, 342-352.

Demers, M., Krause, D. S., Schatzberg, D., Martinod, K., Voorhees, J. R.,

sperm entrapment by NETs (Alghamdi et al., 2004; Alghamdi and Foster, 2005).

The presence of large numbers of NETs directly in the intervillous space in preeclampsia (Gupta et al., 2005a), a crucial interface between mother and fetus, as well as the induction of NETosis by placentally-derived micro-vesicles or cytokines, suggest a role for these entities in this enigmatic disorder (Gupta et al., 2007).

Although still in the realm of speculation, new evidence implicating PMNs activation in fetal loss induced by auto-antibodies (Salmon and Girardi, 2008) or infectious agents (Robbins and Bakardjiev, 2012), may involve the occurrence of NETs.

Fuchs, T. A., et al. (2012). Cancers predispose neutrophils to release extracellular DNA traps that contribute to cancer-associated thrombosis. Proc. Natl. Acad. Sci. U.S.A. 109, 13076-13081.

Fuchs, T. A., Abed, U., Goosmann, C., Hurwitz, R., Schulze, I., Wahn, V., et al. (2007). Novel cell death program leads to neutrophil extracellular traps. J. Cell Biol. 176, 231-241.

Fuchs, T. A., Brill, A., Duerschmied, D., Schatzberg, D., Monestier, M., Myers, D. D. Jr., et al. (2010). Extracellular DNA traps promote thrombosis. Proc. Natl. Acad. Sci. U.S.A. 107, 15880-15885.

Fuchs, T. A., Brill, A., and Wagner, D. D. (2012). Neutrophil extracellular trap (NET) impact on deep vein thrombosis. Arterioscler. Thromb. Vasc. Biol. 32, 1777-1783.

Germain, S. J., Sacks, G. P., Sooranna, S. R., Sargent, I. L., and Redman, C. W. (2007). Systemic inflammatory priming in normal pregnancy and preeclampsia: the role of circulating syncytiotrophoblast microparticles. J. Immunol. 178, 5949-5956.

Girardi, G. (2011). Role of tissue factor in feto-maternal development: a xiphos. J. Thromb. Haemost. 9, 250-256.

Girardi, G., Berman, J., Redecha, P., Spruce, L., Thurman, J. M., Kraus, D., et al. (2003). Complement C5a receptors and neutrophils mediate fetal injury in the antiphospholipid syndrome. J. Clin. Invest. 112, 1644-1654.

Granne, I., Southcombe, J. H., Snider, J. V., Tannetta, D. S., Child, T., Redman, C. W., et al. (2011). ST2 and IL-33 in pregnancy and preeclampsia. PLoS ONE 6:e24463. doi: 10.1371/journal.pone.0024463

Gupta, A., Hasler, P., Gebhardt, S., Holzgreve, W., and Hahn, S. (2006a). Occurrence of neutrophil extracellularsatztraps, DNA (NETs) in pre-eclampsia: a link with elevated levels of cell-free DNA? Ann N.Y. Acad. Sci. 1075, 118-122.

Gupta, A. K., Gebhardt, S., Hillermann, R., Holzgreve, W., and Hahn, S. 
(2006b). Analysis of plasma elastase levels in early and late onset preeclampsia. Arch. Gynecol. Obstet. 273, 239-242.

Gupta, A. K., Hasler, P., Holzgreve, W., Gebhardt, S., and Hahn, S. (2005a). Induction of neutrophil extracellular DNA lattices by placental microparticles and IL-8 and their presence in preeclampsia. Hum. Immunol. 66, 1146-1154.

Gupta, A. K., Rusterholz, C., Holzgreve, W., and Hahn, S. (2005b). Syncytiotrophoblast micro-particles do not induce apoptosis in peripheralsatzlymphocytes, $\mathrm{T}$, but differ in their activity depending on the mode of preparation. J. Reprod. Immunol. 68, 15-26.

Gupta, A. K., Hasler, P., Holzgreve, W., and Hahn, S. (2007). Neutrophil NETs: a novel contributor to preeclampsia-associated placental hypoxia? Semin. Immunopathol. 29, 163-167.

Gupta, A. K., Joshi, M. B., Philippova, M., Erne, P., Hasler, P., Hahn, S., et al. (2010). Activated endothelial cells induce neutrophil extracellular traps and are susceptible to NETosis-mediated cell death. FEBS Lett. 584, 3193-3197.

Gupta, G. K., and Bianchi, D. W. (1997). DNA diagnosis for the practicing obstetrician. Obstet. Gynecol. Clin. North Am. 24, 123-142.

Hahn, S., Gupta, A. K., Troeger, C., Rusterholz, C., and Holzgreve, W. (2006). Disturbances in placental immunology: ready for therapeutic interventions? Springer Semin. Immunopathol. 27, 477-493.

Hahn, S., Huppertz, B., and Holzgreve, W. (2005). Fetal cells and cell free fetal nucleic acids in maternal blood: new tools to study abnormal placentation? Placenta 26, 515-526.

Halim, A., Kanayama, N., El Maradny, E., Maehara, K., Bhuiyan, A. B., and Terao, T. (1996). Correlated plasma elastase and sera cytotoxicity in eclampsia. A possible role of endothelin-1 induced neutrophil activation in preeclampsiaeclampsia. Am. J. Hypertens. 9, 33-38.

Higuchi, D. A., Wun, T. C., Likert, K. M., and Broze, G. J. Jr. (1992). The effect of leukocyte elastase on tissue factor pathway inhibitor. Blood 79, 1712-1719.

Holers, V. M., Girardi, G., Mo, L., Guthridge, J. M., Molina, H., Pierangeli, S. S., et al. (2002). Complement C3 activation is required for antiphospholipid antibody-induced fetal loss. J. Exp. Med. 195, 211-220.
Huppertz, B. (2008). The anatomy of the normal placenta. J. Clin. Pathol. 61, 1296-1302.

Katila, T. (2012). Post-mating inflammatory responses of the uterus. Reprod. Domest. Anim. 47(Suppl. 5), 31-41.

King, A. E., Critchley, H. O., and Kelly, R. W. (2003a). Innate immune defences in the human endometrium. Reprod. Biol. Endocrinol. 1, 116.

King, A. E., Critchley, H. O., Sallenave, J. M., and Kelly, R. W. (2003b). Elafin in human endometrium: an antiprotease and antimicrobial molecule expressed during menstruation. J. Clin. Endocrinol. Metab. 88, 4426-4431.

Kitzmiller, J. L., and Benirschke, K. (1973). Immunofluorescent study of placental bed vessels in pre-eclampsia of pregnancy. Am. J. Obstet. Gynecol. 115, 248-251.

Knowles, H., Heizer, J. W., Li, Y., Chapman, K., Ogden, C. A., Andreasen, K., et al. (2011). Transient Receptor Potential Melastatin 2 (TRPM2) ion channel is required for innate immunity against Listeria monocytogenes. Proc. Natl. Acad. Sci. U.S.A. 108, 11578-11583.

Leung, T. N., Zhang, J., Lau, T. K., Chan, L. Y., and Lo, Y. M. (2001). Increased maternal plasma fetal DNA concentrations in women who eventually develop preeclampsia. Clin. Chem. 47, 137-139.

Lynch, A. M., and Salmon, J. E. (2010). Dysregulated complement activation as a common pathway of injury in preeclampsia and other pregnancy complications. Placenta 31, 561-567.

Marcos, V., Nussbaum, C., Vitkov, L., Hector, A., Wiedenbauer, E. M., Roos, D., et al. (2009). Delayed but functional neutrophil extracellular trap formation in neonates. Blood 114, 4908-4911. author reply: 4911-4912.

Massberg, S., Grahl, L., von Bruehl, M. L., Manukyan, D., Pfeiler, S., Goosmann, C., et al. (2010). Reciprocal coupling of coagulation and innate immunity via neutrophil serine proteases. Nat. Med. 16, 887-896.

Mehta, R., and Petrova, A. (2006). Intrauterine neutrophil activation is associated with pulmonary haemorrhage in preterm infants. Arch. Dis. Child. Fetal Neonatal Ed. 91, F415-F418.

Mellembakken, J. R., Aukrust, P., Olafsen, M. K., Ueland, T., Hestdal, K., and Videm, V. (2002). Activation of leukocytes during the uteroplacental passage in preeclampsia. Hypertension 39 155-160.

Messerli, M., May, K., Hansson, S. R., Schneider, H., Holzgreve, W., Hahn, S., et al. (2010). Feto-maternal interactions in pregnancies: placental microparticles activate peripheral blood monocytes. Placenta 31, 106-112.

Nathan, C. (2006). Neutrophils and immunity: challenges and opportunities. Nat. Rev. Immunol. 6, 173-182.

Neeli, I., Khan, S. N., and Radic, M. (2008). Histone deimination as a response to inflammatory stimuli in neutrophils. J. Immunol. 180, 1895-1902.

Papayannopoulos, V., Metzler, K. D., Hakkim, A., and Zychlinsky, A. (2010). Neutrophil elastase and myeloperoxidase regulate the formation of neutrophil extracellular traps. J. Cell Biol. 191, 677-691.

Pierangeli, S. S., Girardi, G., VegaOstertag, M., Liu, X., Espinola, R. G., and Salmon, J. (2005). Requirement of activation of complement C3 and C5 for antiphospholipid antibodymediated thrombophilia. Arthritis Rheum. 52, 2120-2124.

Pijnenborg, R., Vercruysse, L., and Brosens, I. (2011). Deep placentation. Best practice and research. Clin. Obstet. Gynaecol. 25, 273-285.

Plow, E. F. (1980). The major fibrinolytic proteases of human leukocytes. Biochim. Biophys. Acta 630, 47-56.

Powe, C. E., Levine, R. J., and Karumanchi, S. A. (2011). Preeclampsia, a disease of the maternal endothelium: the role of antiangiogenic factors and implications for later cardiovascular disease. Circulation 123, 2856-2869.

Rana, S., Powe, C. E., Salahuddin, S., Verlohren, S., Perschel, F. H., Levine, R. J., et al. (2012). Angiogenic factors and the risk of adverse outcomes in women with suspected preeclampsia. Circulation 125, 911-919.

Redecha, P., Tilley, R., Tencati, M., Salmon, J. E., Kirchhofer, D., Mackman, N., et al. (2007). Tissue factor: a link between $\mathrm{C} 5 \mathrm{a}$ and neutrophil activation in antiphospholipid antibody induced fetal injury. Blood 110, 2423-2431.

Redman, C. W. (1997). Cytotrophoblasts: masters of disguise. Nat. Med. 3, 610-611.

Redman, C. W., and Sargent, I. L. (2005). Latest advances in understanding preeclampsia. Science 308, 1592-1594.
Redman, C. W., and Sargent, I. L. (2010). Immunology of preeclampsia. Am. J. Reprod. Immunol. 63, 534-543.

Robbins, J. R., and Bakardjiev, A. I. (2012). Pathogens and the placental fortress. Curr. Opin. Microbiol. 15, 36-43.

Roberts, J. M., and Hubel, C. A. (2009). The two stage model of preeclampsia: variations on the theme. Placenta 30(Suppl. A), S32-S37.

Roberts, J. M., and Redman, C. W. (1993). Pre-eclampsia: more than pregnancy-induced hypertension. Lancet 341, 1447-1451.

Rusterholz, C., Hahn, S., and Holzgreve, W. (2007). Role of placentally produced inflammatory and regulatory cytokines in pregnancy and the etiology of preeclampsia. Semin. Immunopathol. 29, 151-162.

Sacks, G. P., Studena, K., Sargent, K., and Redman, C. W. (1998). Normal pregnancy and preeclampsia both produce inflammatory changes in peripheral blood leukocytes akin to those of sepsis. Am. J. Obstet. Gynecol. 179, 80-86.

Saffarzadeh, M., Juenemann, C., Queisser, M. A., Lochnit, G., Barreto, G., Galuska, S. P., et al. (2012). Neutrophil extracellular traps directly induce epithelial and endothelial cell death: a predominant role of histones. PLOS ONE 7:e32366. doi: 10.1371/journal.pone.0032366

Salamonsen, L. A., and Lathbury, L. J. (2000). Endometrial leukocytes and menstruation. Hum. Reprod. Update 6, 16-27.

Salamonsen, L. A., Zhang, J., Hampton, A., and Lathbury, L. (2000). Regulation of matrix metalloproteinases in human endometrium. Hum. Reprod. 15(Suppl. 3), 112-119.

Salmon, J. E., and Girardi, G. (2008). Antiphospholipid antibodies and pregnancy loss: a disorder of inflammation. J. Reprod. Immunol. 77, 51-56.

Sasaki, S., Nagata, K., and Kobayashi, Y. (2009). Regulation of the estrous cycle by neutrophil infiltration into the vagina. Biochem. Biophys. Res. Commun. 382, 35-40.

Sasaki, S., Tamaki, Y., Nagata, K., and Kobayashi, Y. (2011). Regulation of the estrous cycle by neutrophils via opioid peptides. J. Immunol. 187, 774-780.

Semeraro, F., Ammollo, C. T., Morrissey, J. H., Dale, G. L., Friese, P., Esmon, N. L., et al. (2011). Extracellular histones promote 
thrombin generation through platelet-dependent mechanisms: involvement of platelet TLR2 and TLR4. Blood 118, 1952-1961.

Smith, J. M., Shen, Z., Wira, C. R., Fanger, M. W., and Shen, L. (2007). Effects of menstrual cycle status and gender on human neutrophil phenotype. Am. J. Reprod. Immunol. 58, 111-119.

Strzemienski, P. J. (1989). Effect of bovine seminal plasma on neutrophil phagocytosis of bull spermatozoa. J. Reprod. Fertil. 87, 519-528.

Strzemienski, P. J., Dyer, R. M., Sertich, P. L., Garcia, M. C., and Kenney, R. M. (1987). Bactericidal activity of peripheral blood neutrophils during the oestrous cycle and early pregnancy in the mare. J. Reprod. Fertil. 80, 289-293.

von Bruhl, M. L., Stark, K., Steinhart,

A., Chandraratne, S., Konrad, I., Lorenz, M., et al. (2012). Monocytes, neutrophils, and platelets cooperate to initiate and propagate venous thrombosis in mice in vivo. J. Exp. Med. 209, 819-835.
Wang, Y., Li, M., Stadler, S., Correll, S., Li, P., Wang, D., et al. (2009). Histone hypercitrullination mediates chromatin decondensation and neutrophil extracellular trap formation. J. Cell Biol. 184, 205-213.

Weiler, H. (2008). Tracing the molecular pathogenesis of antiphospholipid syndrome. J. Clin. Invest. 118, 3276-3278.

Wilkinson, H. (2011). Saving mothers' lives. Reviewing maternal deaths to make motherhood safer: 2006-2008. BJOG 118, 1402-1403. discussion: 1403-1404.

Wood, G. A., Fata, J. E., Watson, K. L., and Khokha, R. (2007). Circulating hormones and estrous stage predict cellular and stromal remodeling in murine uterus. Reproduction 133, 1035-1044.

Yost, C. C., Cody, M. J., Harris, E. S., Thornton, N. L., McInturff, A. M., Martinez, M. L., et al. (2009). Impaired neutrophil extracellular trap (NET) formation: a novel innate immune deficiency of human neonates. Blood 113, 6419-6427.

Zhong, X. Y., Gebhardt, S., Hillermann, R., Tofa, K. C.,
Holzgreve, W., and Hahn, S. (2005a). Parallel assessment of circulatory fetal DNA and corticotropin-releasing hormone mRNA in early- and late-onset preeclampsia. Clin. Chem. 51, 1730-1733.

Zhong, X. Y., Gebhardt, S., Hillermann, R., Tofa, K. C., Holzgreve, W., and Hahn, S. (2005b). Circulatory nucleosome levels are significantly increased in early and late-onset preeclampsia. Prenat. Diagn. 25, 700-703.

Zhong, X. Y., Holzgreve, W., and Hahn, S. (2001a). Circulatory fetal and maternal DNA in pregnancies at risk and those affected by preeclampsia. Ann. N.Y. Acad. Sci. 945, 138-140.

Zhong, X. Y., Laivuori, H., Livingston, J. C., Ylikorkala, O., Sibai, B. M., Holzgreve, W., et al. (2001b). Elevation of both maternal and fetal extracellular circulating deoxyribonucleic acid concentrations in the plasma of pregnant women with preeclampsia. Am. J. Obstet. Gynecol. 184, 414-419.
Conflict of Interest Statement: The authors declare that the research was conducted in the absence of any commercial or financial relationships that could be construed as a potential conflict of interest.

Received: 11 October 2012; paper pending published: 29 October 2012; accepted: 12 November 2012; published online: 27 November 2012.

Citation: Hahn S, Giaglis S, Hoesli $I$ and Hasler P (2012) Neutrophil NETs in reproduction: from infertility to preeclampsia and the possibility of fetal loss. Front. Immun. 3:362. doi: 10.3389/ fimmu.2012.00362

This article was submitted to Frontiers in Molecular Innate Immunity, a specialty of Frontiers in Immunology.

Copyright (C) 2012 Hahn, Giaglis, Hoesli and Hasler. This is an openaccess article distributed under the terms of the Creative Commons Attribution License, which permits use, distribution and reproduction in other forums, provided the original authors and source are credited and subject to any copyright notices concerning any third-party graphics etc. 